\title{
INDOOR NAVIGATION AND MAPPING: PERFORMANCE ANALYSIS OF UWB-BASED PLATFORM POSITIONING
}

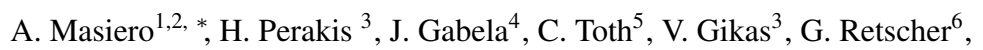 \\ S. Goel ${ }^{7}$, A. Kealy ${ }^{8}$, Z. Koppányi ${ }^{9}$, W. Błaszczak-Bak ${ }^{10}$, Y. Li $^{11}$, D. Grejner-Brzezinska ${ }^{12}$ \\ ${ }^{1}$ Dept. of Civil and Environmental Engineering, University of Florence, Italy - andrea.masiero@unifi.it \\ 2 Interdept. Research Center of Geomatics (CIRGEO), University of Padova, Italy - masiero@dei.unipd.it \\ ${ }^{3}$ School of Rural and Surveying Engineering, National Technical University of Athens, Athens, Greece \\ ${ }^{4}$ Department of Electrical and Electronic Engineering, The University of Melbourne, Australia \\ 5 Department of Civil, Environmental and Geodetic Engineering, The Ohio State University, Columbus, Ohio, USA \\ ${ }^{6}$ Department of Geodesy and Geoinformation, TU Wien-Vienna University of Technology, Vienna, Austria \\ ${ }^{7}$ Department of Civil Engineering, Indian Institute of Technology, Kanpur, India \\ ${ }^{8}$ Department of Geospatial Science, RMIT University, Melbourne, Australia \\ ${ }^{9}$ Leica Geosystems, Heerbrugg, Switzerland \\ ${ }^{10}$ Institute of Geodesy of the University of Warmia and Mazury, Olsztyn, Poland \\ 11 SMART Infrastructure Facility, University of Wollongong, Australia \\ 12 College of Engineering, The Ohio State University, Columbus, Ohio, USA
}

KEY WORDS: Indoor positioning, Sensor fusion, UWB, SLAM, Deep Learning

\begin{abstract}
:
The increasing demand for reliable indoor navigation systems is leading the research community to investigate various approaches to obtain effective solutions usable with mobile devices. Among the recently proposed strategies, Ultra-Wide Band (UWB) positioning systems are worth to be mentioned because of their good performance in a wide range of operating conditions. However, such performance can be significantly degraded by large UWB range errors; mostly, due to non-line-of-sight (NLOS) measurements. This paper considers the integration of UWB with vision to support navigation and mapping applications. In particular, this work compares positioning results obtained with a simultaneous localization and mapping (SLAM) algorithm, exploiting a standard and a Time-of-Flight (ToF) camera, with those obtained with UWB, and then with the integration of UWB and vision. For the latter, a deep learning-based recognition approach was developed to detect UWB devices in camera frames. Such information is both introduced in the navigation algorithm and used to detect NLOS UWB measurements. The integration of this information allowed a $20 \%$ positioning error reduction in this case study.
\end{abstract}

\section{INTRODUCTION}

The development of GNSS (Global Navigation Satellite Systems) allowed any person with a proper receiver to assess his/her own position in real time at almost any outdoor location worldwide. Since nowadays GNSS receivers are embedded in most of the smart mobile devices (such as smartphones) available on the market, billions of users can easily access this technology. The availability of the above-mentioned technologies and the huge base of potential users have motivated the development of many civilian applications, and in particular, many location based services (LBS), which take advantage of the worldwide use of mobile devices with GNSS receivers to provide services based on the knowledge of user's position. The global LBS market quickly increased its size in the last decade, and it is still expected to grow at $36 \%$ compound annual growth rate till 2023 (Market Reports World, 2019).

Despite an unequaled importance of GNSS in outdoor positioning, it is quite unreliable in certain operating conditions, such as indoors and in city centers, close to skyscrapers. Actually, reliable and accurate positioning in GNSS-denied environments is a rather challenging task, which is attracting a lot of attention from the research community. Clearly, such interest is also motivated by the LBS market Furthermore, on average, people

\footnotetext{
* Corresponding author.
}

spend approximately 90 percent of their time indoors (Scribner, 2018), and thus, a significant increase of the LBS market is expected to come from an extension of LBS to indoors, which is obviously possible only if an effective ubiquitous navigation strategy is developed; e.g., able to properly deal with indoor navigation and with the transition between outdoors and indoors positioning.

A commonly accepted approach to such aim is the integration od information provided by several sensors in order to improve the overall positioning ability of the considered mobile device. Sensors typically considered for such sensor fusion often include: inertial sensors, radio signals (e.g. WiFi, Bluetooth, UWB), and cameras.

Since MEMS (micro-electromechanical system) based inertial sensors are extremely cheap, e.g. tens of dollars, they are embedded in almost all smart mobile devices currently on the market. Furthermore, they can be used in practically all the possible working conditions in a dead reckoning positioning strategy, independently from any other sensor. Such characteristics make them an essential component of any integrated positioning system. However, any kind of dead reckoning strategy implemented by using only such cheap sensors rapidly leads to a drift of the position estimates with respect to the ground truth, hence any reliable navigation strategy should use them in combination 
with other sensors (Davidson, Piché, 2016).

Visual odometry is a widely investigated research field concerning the use of vision (e.g. cameras) to determine user's device relative movements. Furthermore, vision can also be used to produce 3D information: the approach combining positioning with producing spatial information, simultaneous localization and mapping (SLAM), is also widely used, for both such purposes. Despite the good performance potential of such visionbased approaches, it is well known (IPIN, 2019, Wang et al., 2019) that real-time image processing can be a quite computational power demanding task, hence it can lead to a relatively fast draining of the battery of mobile devices.

The use of radio signals is also very often considered for indoor positioning; in particular, WiFi has been widely considered in the literature (Casari et al., 2009, Sakr, El-Sheimy, 2017, Retscher et al., 2019), mostly because of its frequent availability in most of the buildings. Among the radio signals, Ultra-Wide Band (UWB) has recently attracted the attention of several researchers working on indoor positioning thanks to its better multipath resilience with respect to other radio signal positioning technologies, its low interference with other existing system, and low power requirements. UWB systems based on two-way time of flight (TW-ToF) typically provide quite accurate ranging measurements; the accuracy of the ranging measurements obtained in direct line of sight (LOS) is typically at decimeter level, whereas obstructions (i.e. non-line-of-sight (NLOS) measurements) can lead to a significant degradation of the range measurement accuracy (Dabove et al., 2018).

Furthermore, most of the commercial UWB positioning systems are based just on the use of a set of UWB devices fixed at known positions, typically named anchors, but it is clear that communication and relative range measurements between moving devices can also be exploited for improving users' position estimates in a cooperative positioning strategy (Seco, Jiménez, 2018, Kealy et al., 2015, Gabela et al., 2019).

Given the low cost of certain UWB transceivers, e.g. Decawave, the next generation of smartphones might be provided with such technology, opening the possibility to the development of a positioning strategy based on UWB relative range measurements between moving devices, reducing the need for an external UWB infrastructure (Sakr et al., 2020).

Motivated by the previously mentioned considerations on the relevance of NLOS measurements in UWB systems, this work aims at investigating a suitable strategy for improving UWB performance in realistic indoor working conditions. In particular, this work aims at assessing the performance obtained by detecting and partially compensating NLOS UWB measurements by means of an integrated UWB and vision positioning system. Given the high power consumption related to the use of vision algorithms, the vision system is used only to periodically correct the estimated device position. The correction is based on the deep-learning recognition of UWB anchors in the camera images, and then applying the corresponding positional information in the navigation algorithm. Obtained results are compared with those of a SLAM algorithm, which is used for providing both a reference trajectory and a $3 \mathrm{D}$ reconstruction of the test area.

Experimental results are based on tests carried out in a 4-storey University building at Ohio State University, by using 30 UWB anchors (Time Domain and Pozyx) and 4 moving users, and on a 3-storey building of the University of Padua (Italy), by using 12 anchors (Pozyx) and a moving user, collecting data simultaneously also with a standard (RGB) and Time-of-Flight (ToF) camera.

\section{EFFECT OF NLOS UWB RANGE MEASUREMENTS ON POSITIONING}

UWB positioning is based on the use of a set of anchors, fixed at known positions and rovers, moving in the area of interest. The aim of this kind of systems is typically to provide reliable estimates of the rovers' movement, the computation is based on the anchor-rover range measurements acquired by the rovers by periodically communicating with the anchors.

UWB systems typically allow to obtain accurate positioning with error at decimeter level when LOS measurements are available, whereas the system performance usually degrades in presence of NLOS measurements.

Motivated by the above consideration, a number of methods have been formulated in the literature for NLOS measurement detection (Marano et al., 2010), mostly related either to the analysis of the characteristics of the received signal (Yu et al., 2018, Yang, 2018) or the geometric consistency of the position estimate. Dynamically adapting the measurement variance in the tracking algorithm has also been considered to reduce the effect of unreliable measurements (Pointon et al., 2019).

A number of NLOS issues are caused by objects which are part of the static environment in the area, such as walls, furniture. The impact of static, spatially inhomogeneous structures on RF signal propagation can be assessed by means of a calibration procedure, which typically ensures also a positioning performance improvement in the areas where NLOS issues are more apparent (Retscher et al., 2020).

Fig. 1 shows the UWB ranging error reduction in terms of cumulative distribution function, obtained after calibration of a 14-anchor Pozyx system in a 4-storey University building at Ohio State University.

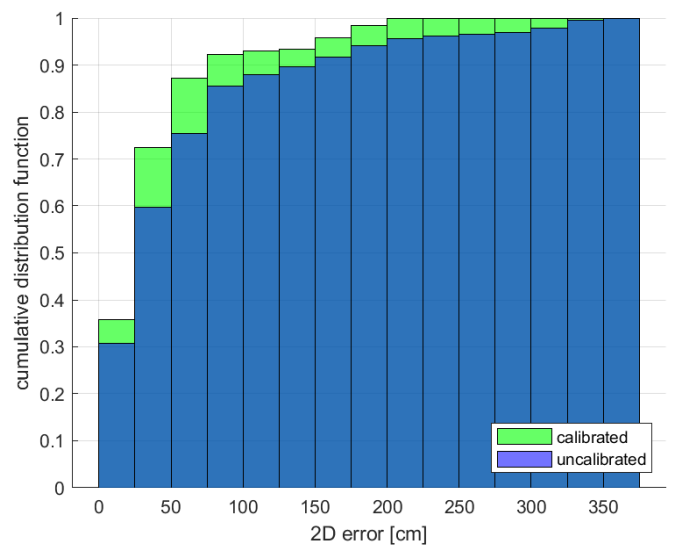

Figure 1. Example of distribution of uncalibrated and calibrated Pozyx UWB ranges.

\section{INTEGRATION OF UWB WITH VISION}

Despite of proper calibration of the UWB system used to improve the positioning results, such calibration should be updated any time when a change occurs in the environment. As 
a different approach to calibration, here we propose the integration of UWB with vision to improve the UWB system performance, while reducing the need for calibration and thus the update of the UWB system.

Furthermore, since the execution of vision algorithms on a mobile device may quickly drain off the battery, in the proposed approach, vision algorithms are not continuously running; they are called periodically at certain time instants, aiming at assessing and compensating the UWB positioning error.

The rationale for using an RGB-ToF camera to determine the presence of UWB anchors in the acquired frames is that if detected, use them as additional information to aid the navigation algorithm.

The anchor detection strategy is as follows. First, the depth frame acquired by the ToF camera is converted to a point cloud in a local reference system. Then, horizontal planes are detected in the obtained point cloud (e.g., detecting ground and/or ceiling) and thus the point cloud is vertically leveled accordingly. Next, a set of candidate anchors is determined by searching small connected regions of points close to the ground, where anchors are assumed to be positioned in this case study . It is worth to notice that such method can clearly be extended to more general anchor positions, provided that they are well distinguishable in the point clouds. The set of candidate anchor points are backprojected on the RGB image, and their neighborhood is used as input for a deep learning-based classifier, which aims at distinguishing anchors from other objects. Then, the detected anchors are associated to real ones based on the similarity between measured UWB ranges and distances measured by the ToF camera. Finally, detected anchors can be used as control points in the navigation algorithm.For example, if two or more anchors are detected in a frame, a $2 \mathrm{D} / 3 \mathrm{D}$ position fix can be obtained, otherwise a just a range measurement is provided by the vision system. In the results reported in the following, this approach will be shortly named "uwb+rec", where "rec" stands for anchor recognition.

Fig. 2 shows an example of detected anchor (green box), and candidates not classified as anchors (red boxes). Such candidates are sometimes associated to outlier 3D points, found in the point cloud.

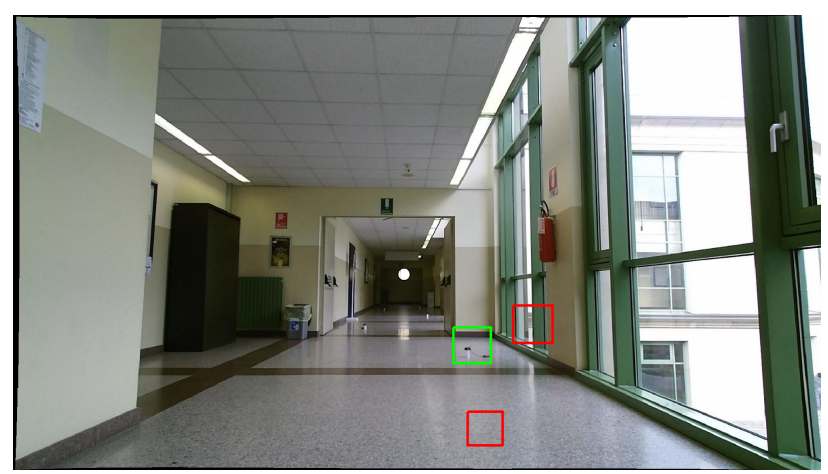

Figure 2. Example of Pozyx UWB anchor recognition: correctly classified anchor (green box), examples of candidates not classified as anchors (red boxes).

It is worth to notice that when an anchor provides an observation in NLOS, its successive measurements are also often in NLOS as well. Furthermore, the associated error may vary in such set of measurements, depending on the user movements and on the environment and anchor geometric configuration. In practice, modeling such error without a proper calibration process might be quite challenging. Nevertheless, detecting and discarding NLOS observations can often be sufficient for reaching a notable positioning performance improvement.

Given the above consideration, in the approach named hereafter "uwb+rec+NLOS" NLOS observation detection is performed when a position fix from the vision system is available. Note that position fix uncertainty is usually very small, hence detecting large differences between measured UWB ranges and distances from the estimated position to the corresponding anchors can be considered as a reliable way to identify NLOS observations. Once a measurement is detected in NLOS, its corresponding anchor is discarded by the navigation algorithm in a spatial neighborhood (circular, with diameter 4 meters in our tests) of the position where NLOS condition has been detected.

\section{SLAM FOR POSITIONING AND MAPPING}

The presence of a vision system in the navigation device enables the use of such system for both navigation and mapping purposes. More specifically, a simultaneous localization and mapping (SLAM, (Leonard, Durrant-Whyte, 1991)) algorithm can be conveniently used to reliably determine both the current position of the device and to build a map of the area around the vision acquisition system under normal conditions.

In this paper a SLAM algorithm was developed to exploit both the information provided by the RGB and ToF cameras. To be more specific, the last available frame is just compared with the previous one in order to determine the relative movement between them. Clearly, such approach is suboptimal, however it reduces the computational time (limiting the information to be extracted and compared) and the quantity of information.

Hence, despite being suboptimal, such approach is quite reasonable in navigation applications, where keeping all the previously acquired spatial information about the environment is not required. Alternatively, other approaches might also be considered to ease the use of spatial information collected in previous frames (Forstner, Khoshelham, 2017, Nguyen et al., 2007).

If UWB anchors are present in the area of interest, their location data can be exploited to support the SLAM reconstruction and localization information in the UWB reference system. The anchor detection algorithm can be executed in parallel to SLAM, on the overall point cloud generated with the SLAM algorithm, to determine the anchor positions. Then, the detected anchors can be used as ground control to change the reference system of the SLAM point cloud from local to that of the UWB system. The fitting error between the measured (ground truth) anchor positions and those obtained with the SLAM can be used to assess the level of reliability of the SLAM positioning system (and, partially, for the mapping).

\section{RESULTS}

SLAM is used for localization and 3D reconstruction of a corridor of a building of the University of Padua, Italy. UWB anchors were distributed over the area of interest (Fig. 3), hence the SLAM results can be expressed in the UWB reference system, as described in the previous section. The fitting error resulting from the conversion of the mapping reference system to 
that of the UWB system is about $4.4 \mathrm{~cm}$, which is acceptable, given the typical error associated to ToF measurements (Lachat et al., 2015, Masiero et al., 2019) and the distance traveled (approximately $45 \mathrm{~m}$ ). As previously mentioned, such results provide a rough assessment of the SLAM positioning accuracy. The top view of the 3D reconstruction of the considered corridor is shown in Fig. 4.

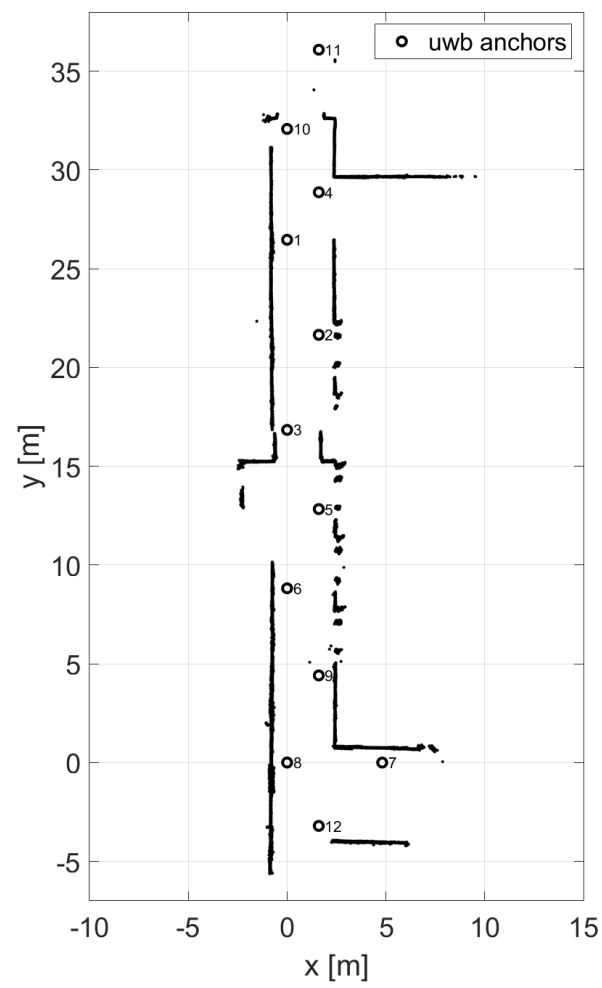

Figure 3. UWB anchor distribution.

Given the fairly high SLAM reliability in this case study, its navigation (and mapping) solution is used as reference to validate the other positioning approaches in the following.

More specifically, the results of three positioning methods are compared:

- UWB positioning, alone (see Fig. 5).

- "uwb+rec": UWB positioning combined with the previously described UWB anchors recognition, used to correct the UWB error (Fig. 6)

- "uwb+rec+NLOS": UWB positioning combined with UWB anchor recognition, and with the detection of NLOS measurements when a position fix is available. Anchors providing NLOS observation are temporarily discarded in the navigation algorithm (Fig. 7).

Numerical positioning results of the considered approaches are reported in Table 1. Such positioning errors were evaluated on a set of 41 locations, using SLAM estimated positions as reference (shown as red circular marks in Fig. 5, 6 and 7). To be more precise, "uwb+rec" and "uwb+rec+NLOS" errors are evaluated before applying any correction provided by the vision system, whereas "uwb+rec (post)" "uwb+rec+NLOS (post)" are evaluated in the tracking step just after such corrections.

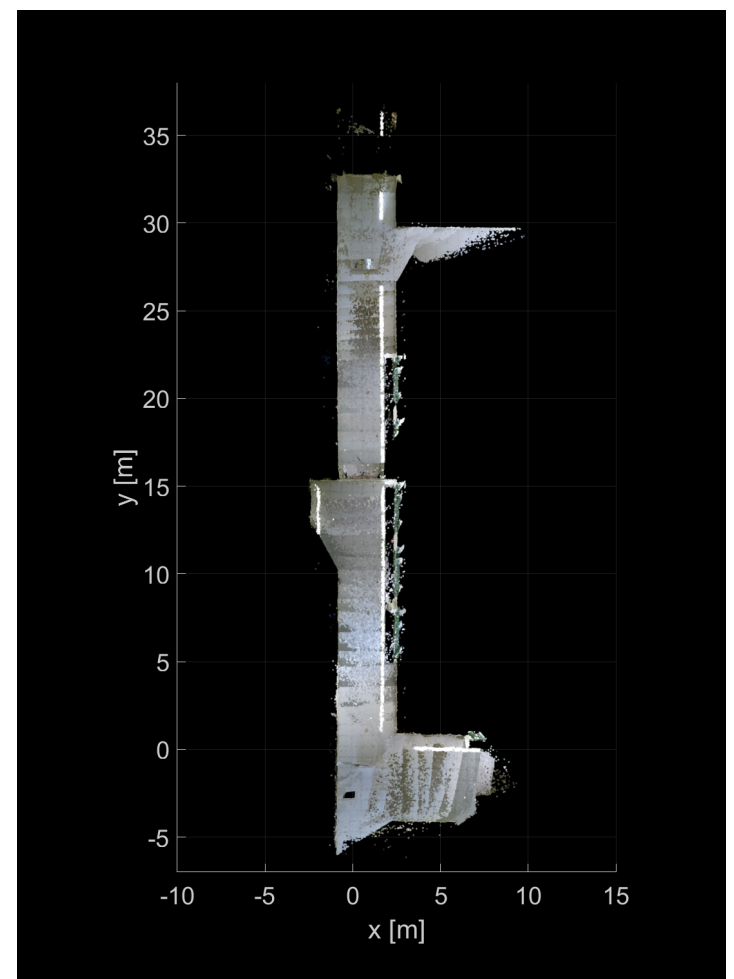

Figure 4. Top view of the 3D reconstruction of the considered corridor.

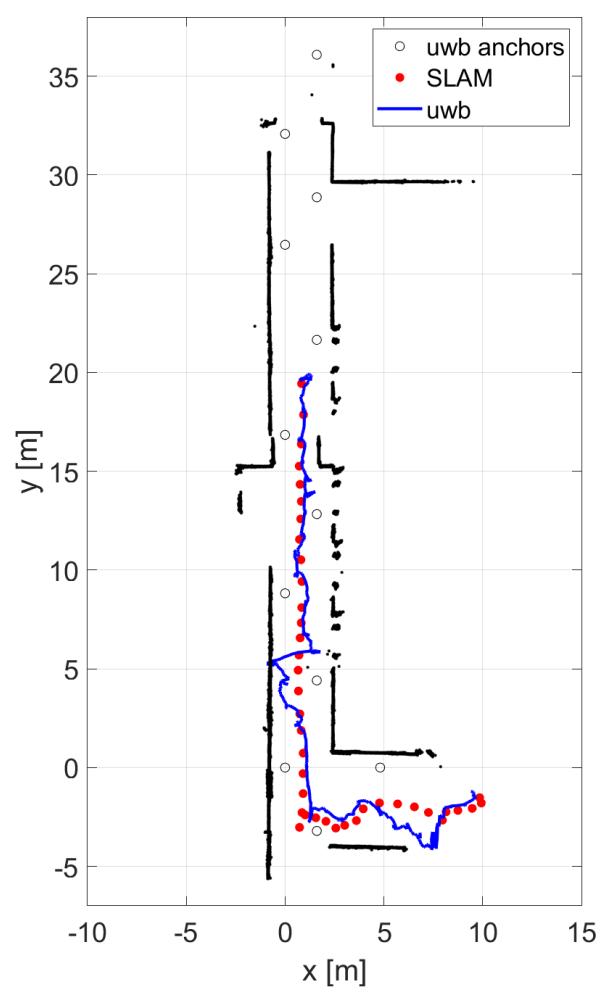

Figure 5. UWB positioning.

\section{DISCUSSION}

Fig. 5 shows that UWB positioning is very effective for $y \geq 7$ and it is also working fine for $y \leq 2$ and $x \leq 3$. However, two critical areas can be identified (a) for $x \geq 3$ (visible in Fig. 8(a)) 


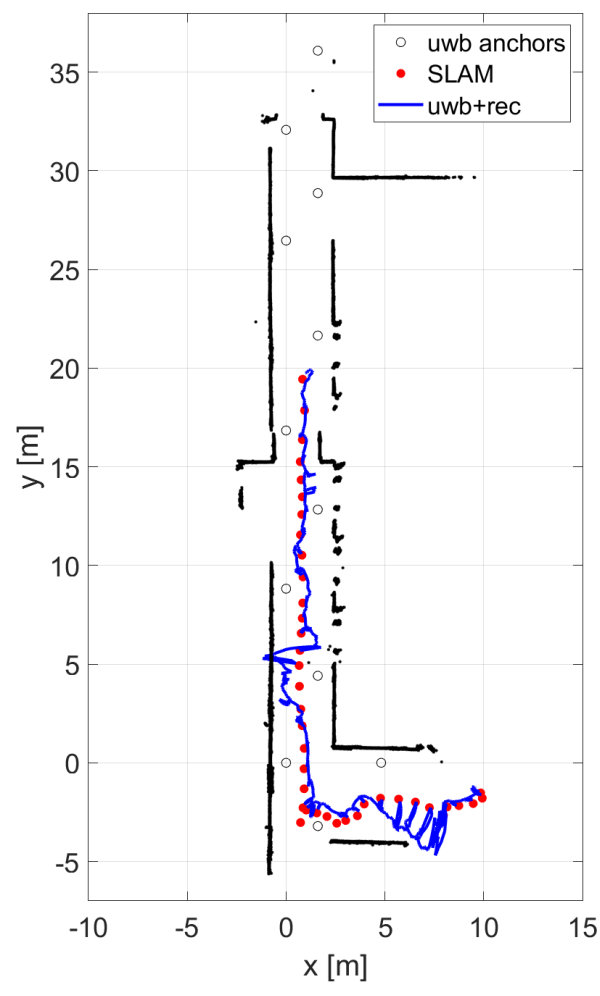

Figure 6. UWB+anchor recognition positioning.

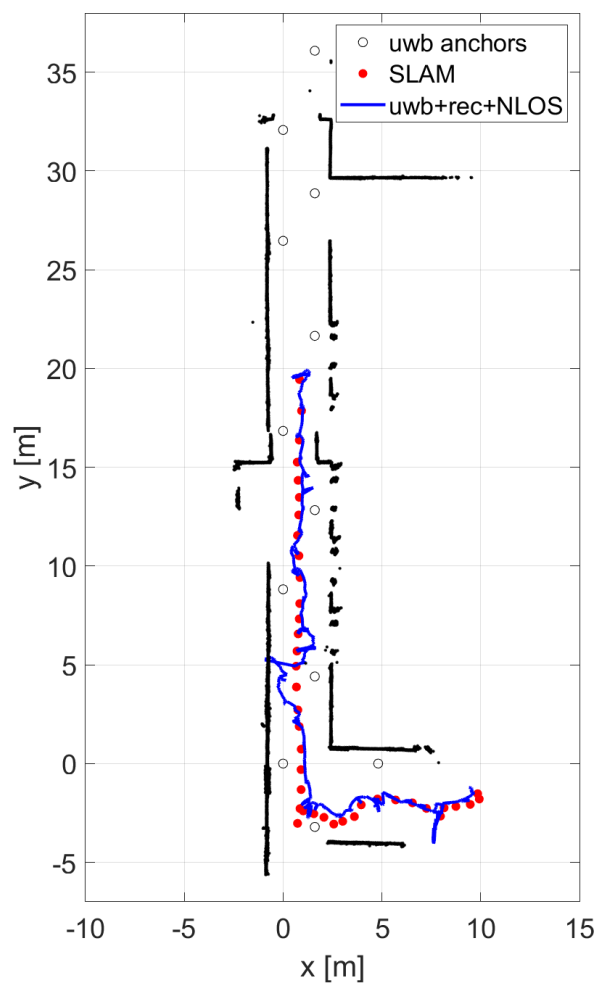

Figure 7. UWB+anchor recognition+NLOS detection positioning.

and (b) for $2 \leq y \leq 3$ (visible in Fig. 8(b)). COMMENT: you may put in circles to better show areas (a) and (b) in the figure.

Bad performance of the UWB system in such areas is mostly due to the presence of large range errors due to NLOS measurements provided by anchors 7 and 9 (see Fig. 3 for the anchor
Table 1. 2D positioning performance comparison to SLAM

\begin{tabular}{c|c|c}
\hline Approach & RMSE [cm] & $\max [\mathrm{cm}]$ \\
\hline \hline uwb & 65 & 154 \\
uwb+rec & 65 & 153 \\
uwb+rec (post) & 33 & 119 \\
uwb+rec+NLOS & 52 & 154 \\
uwb+rec+NLOS (post) & 33 & 76 \\
\hline
\end{tabular}

positions).

The use of vision-based corrections, approach "uwb+rec" allows to effectively reduce the position estimation error in area (a). However, the presence of large range errors quite quickly degrade the positioning performance after such corrections. In contrast, determining and temporarily disabling NLOS observations ensures better performance in such areas with the approach "uwb+rec+NLOS".

The performance of the three approaches is quite similar in area (b), because no vision-based position fix was available in this area.

The obtained numerical results confirm the effectiveness of the vision-based corrections ("post" results in Table 1). It is worth to notice that since position fix corrections were available only in part of the considered points of the track, the overall "post" errors were larger than the expected.

Furthermore, the numerical results of the "uwb+rec" approach are very similar to those of the "uwb" one, showing that, since NLOS measurements are typically present in several consecutive measurements, the battery-saving infrequent use of vision corrections does not allow to properly compensate the presence of such NLOS observations. Obviously, it is a tradeoff, and ignoring the battery consideration, the performance can be significantly increased, depending on local circumstances.

In summary, exploiting vision information to determine NLOS observations also led to a notable performance improvement where position fix corrections are available.

\section{CONCLUSIONS}

This paper investigated the integration of UWB with vision to improve navigation accuracy. Given the high power consumption typically associated to vision data processing, vision process was periodically executed to reduce the UWB positioning error.

The error reduction was based on detecting UWB anchors in the RGB-ToF camera frames, and then introducing the corresponding positional information in the navigation solution. Furthermore, due to the availability of this information, NLOS observations were also detected, and consequently removed from the solution.

According to our experimental results, the aiding of UWB by vision can improve the positioning performance; in our case study, a 20\% improvement was achieved, which is a conservative number, as the vision system was not continuously used. 


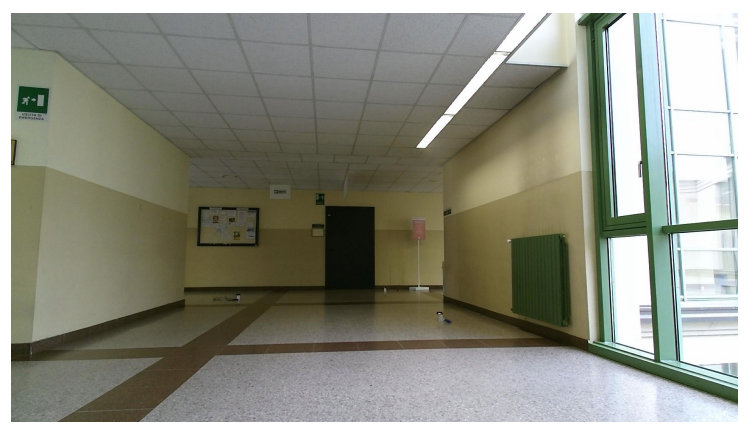

(a)

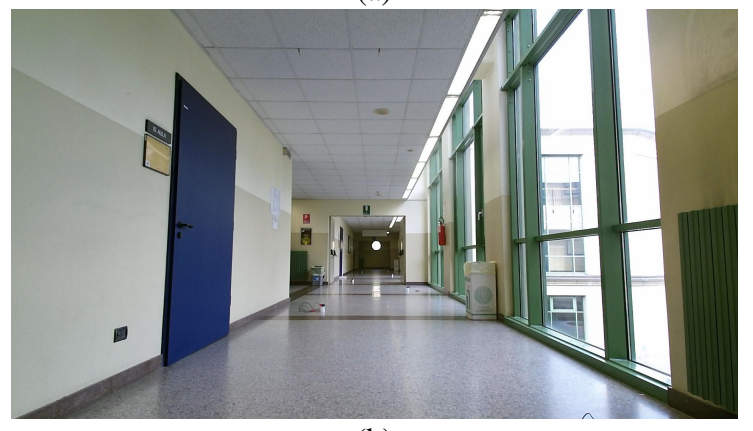

(b)

Figure 8. Critical areas: presence of large UWB range errors due to NLOS measurements.

\section{REFERENCES}

Casari, P., Castellani, A., Cenedese, A., Lora, C., Rossi, M., Schenato, L., Zorzi, M., 2009. The "Wireless Sensor Networks for City-Wide Ambient Intelligence (WISE-WAI)" Project. Sensors, 9(6), 4056-4082.

Dabove, P., Di Pietra, V., Piras, M., Jabbar, A. A., Kazim, S. A., 2018. Indoor positioning using ultra-wide band (uwb) technologies: Positioning accuracies and sensors' performances. 2018 IEEE/ION Position, Location and Navigation Symposium (PLANS), IEEE, 175-184.

Davidson, P., Piché, R., 2016. A survey of selected indoor positioning methods for smartphones. IEEE Communications Surveys \& Tutorials, 19(2), 1347-1370.

Forstner, W., Khoshelham, K., 2017. Efficient and accurate registration of point clouds with plane to plane correspondences. Proceedings of the IEEE International Conference on Computer Vision, 2165-2173.

Gabela, J., Retscher, G., Goel, S., Perakis, H., Masiero, A., Toth, C., Gikas, V., Kealy, A., Koppányi, Z., Błaszczak-Bak, W. et al., 2019. Experimental evaluation of a UWB-based cooperative positioning system for pedestrians in GNSS-denied environment. Sensors, 19(23), 5274.

IPIN, 2019. Video based competition track.

Kealy, A., Retscher, G., Toth, C., Hasnur-Rabiain, A., Gikas, V., Grejner-Brzezinska, D., Danezis, C., Moore, T., 2015. Collaborative navigation as a solution for PNT applications in GNSS challenged environments-report on field trials of a joint FIG/IAG working group. Journal of Applied Geodesy, 9(4), 244-263.

Lachat, E., Macher, H., Landes, T., Grussenmeyer, P., 2015. Assessment and calibration of a RGB-D camera (Kinect v2
Sensor) towards a potential use for close-range 3D modeling. Remote Sensing, 7(10), 13070-13097.

Leonard, J., Durrant-Whyte, H., 1991. Simultaneous map building and localization for an autonomous mobile robot. Intelligent Robots and Systems '91. 'Intelligence for Mechanical Systems, Proceedings IROS '91. IEEE/RSJ International Workshop on, 1442-1447 vol.3.

Marano, S., Gifford, W. M., Wymeersch, H., Win, M. Z., 2010. NLOS identification and mitigation for localization based on UWB experimental data. IEEE Journal on selected areas in communications, 28(7), 1026-1035.

Market Reports World, 2019. Global location-based services market - segmented by location (indoor, outdoor), product (hardware, software, services), applications, technology, end user, and region - growth, trends, and forecast (2018 - 2023).

Masiero, A., Guarnieri, A., Vettore, A., 2019. Assessment of a Portable ToF Camera and Comparison with Smartphone Stereo Vision. ISPRS - International Archives of the Photogrammetry, Remote Sensing and Spatial Information Sciences, XLII2/W17, 187-193. doi.org/10.5194/isprs-archives-XLII-2-W17187-2019.

Nguyen, V., Harati, A., Siegwart, R., 2007. A lightweight slam algorithm using orthogonal planes for indoor mobile robotics. 2007 IEEE/RSJ International Conference on Intelligent Robots and Systems, IEEE, 658-663.

Pointon, H. A., McLoughlin, B. J., Matthews, C., Bezombes, F. A., 2019. Towards a Model Based Sensor Measurement Variance Input for Extended Kalman Filter State Estimation. Drones, 3(1), 19.

Retscher, G., Gikas, V., Hofer, H., Perakis, H., Kealy, A., 2019. Range validation of UWB and Wi-Fi for integrated indoor positioning. Applied Geomatics, 11(2), 187-195.

Retscher, G., Kealy, A., Gabela, J., Li, Y., Goel, S., Toth, C. K., Masiero, A., Błaszczak-Bak, W., Gikas, V., Perakis, H. et al., 2020. A benchmarking measurement campaign in GNSS-denied/challenged indoor/outdoor and transitional environments. Journal of Applied Geodesy, 14(2), 215-229.

Sakr, M., El-Sheimy, N., 2017. Efficient wi-fi signal strength maps using sparse gaussian process models. 2017 International Conference on Indoor Positioning and Indoor Navigation (IPIN), IEEE, 1-8.

Sakr, M., Masiero, A., El-Sheimy, N., 2020. LocSpeck: A Collaborative and Distributed Positioning System for Asymmetric Nodes Based on UWB Ad-Hoc Network and Wi-Fi Fingerprinting. Sensors, 20(1), 78.

Scribner, H., 2018. “indoor generation”: Heres how much time we spend indoors.

Seco, F., Jiménez, A., 2018. Smartphone-based cooperative indoor localization with RFID technology. Sensors, 18(1), 266.

Wang, C., Dai, Y., El-Sheimy, N., Wen, C., Retscher, G., Kang, Z., Lingua, A., 2019. Progress on ISPRS Benchmark on Multisensory Indoor Mapping and Positioning. ISPRS - International Archives of the Photogrammetry, Remote Sensing and Spatial Information Sciences, XLII-2/W13. 
Yang, X., 2018. NLOS mitigation for UWB localization based on sparse pseudo-input Gaussian process. IEEE Sensors Journal, 18(10), 4311-4316.

Yu, K., Wen, K., Li, Y., Zhang, S., Zhang, K., 2018. A novel
NLOS mitigation algorithm for UWB localization in harsh indoor environments. IEEE Transactions on Vehicular Techno$\log y, 68(1), 686-699$. 